\title{
Cardinal Invariants of Strongly Porous Sets
}

\author{
OSVALDO GUZMÁN \\ MICHAEL HRUŠÁK \\ ARTURO MARTÍNEZ-CELIS
}

\begin{abstract}
In this work we study cardinal invariants of the ideal SP of strongly porous sets on ${ }^{\omega} 2$. We prove that $\operatorname{add}(\mathbf{S P})=\omega_{1}, \operatorname{cof}(\mathbf{S P})=\mathfrak{c}$ and that it is consistent that $\operatorname{non}(\mathbf{S P})<\operatorname{add}(\mathcal{N})$, answering questions of Hrušák and Zindulka. We also find a connection between strongly porous sets on ${ }^{\omega} 2$ and the Martin number for $\sigma$-linked partial orders, and we use this connection to construct a model where all the Martin numbers for $\sigma-k$-linked forcings are mutually different.
\end{abstract}

2010 Mathematics Subject Classification 03E17, 28A75 (primary); 03E35, 03E50 (secondary)

Keywords: porous sets, cardinal invariants, ideals, Martin number

\section{Introduction}

In 1967, Dolženko [5] began the study of $\sigma$-porous sets ${ }^{1}$ and since then, many applications have been found. One of these appears in [10], where Preiss and Zajíček proved that, given a Banach space $X$ with a separable dual and a continuous convex function $f$ on $X$, the set of points in which $f$ is not Fréchet differentiable is $\sigma$-porous. Other applications can be found in Belna, Evans and Humke [2], Lindenstrauss and Preiss [7], Lindenstrauss, Preiss and Tišer [8], Reich and Zaslavski [11], Renfro [12] and Zaslavski [19].

We shall study the notion of strong porosity: given a metric space $\langle X, d\rangle$, a subset $A \subseteq X$ is strongly porous if there is a $p>0$ such that for any $x \in X$ and any $0<r<1$, there is $y \in X$ such that $B_{p \cdot r}(y) \subseteq B_{r}(x) \backslash A$. In this paper we will refer to strongly porous sets as porous sets. We shall work mostly with porous sets in ${ }^{\omega_{2}} 2$ : we say that a set $A \subseteq{ }^{\omega} 2$ is $n$-porous if for every $s \in{ }^{<\omega_{2}}$ there is a $t \in{ }^{n} 2$ such that $\left\langle s^{\wedge} t\right\rangle \cap A=\emptyset$. By $s^{\frown} t$ we denote the concatenation of $s$ followed by $t$, and by $\langle s\rangle$ we denote the cone of $s$, that is $\langle s\rangle=\left\{f \in{ }^{\omega} 2: s \subseteq f\right\}$. It is easy to see (see Hrušák and Zindulka [6]) that

\footnotetext{
${ }^{1} \mathrm{~A} \sigma$-(upper/lower) porous set is the countable union of (upper/lower) porous sets
} 
a set $A \subseteq{ }^{\omega} 2$ is porous if and only if there is an $n \in \omega$ such that $A$ is $n$-porous. Zajíček [17] proved that a set $A$ in a metric space $\langle X, d\rangle$ is $\sigma$-porous if and only if it is $\sigma$-lower porous, where $A$ is lower porous if for every $x \in X$ there exist $\rho_{x}>0$ and $r_{x}>0$ such that for any $0 \leq r \leq r_{x}$ there is $y \in X$ such that $B_{\rho_{x} \cdot r}(y) \subseteq B_{r}(x) \backslash A$. Another classical notion of porosity is upper porosity: a set $A$ in a metric space $\langle X, d\rangle$ is upper porous if for every $x \in X$ there is $\rho_{x}>0$ and a sequence $r_{n} \rightarrow 0$ such that for every $n \in \omega$ there is $y_{n} \in X$ such that $B_{\rho_{x} \cdot r_{n}}\left(y_{n}\right) \subseteq B_{r_{n}}(x) \backslash A$. It is easy to see that lower porosity implies upper porosity.

We will denote the $\sigma$-ideal ${ }^{2}$ generated by porous sets on ${ }^{\omega} 2$ by $\mathbf{S P}$, the $\sigma$-ideal generated by $n$-porous sets by $\mathbf{S P}_{n}$, and the $\sigma$-ideal generated by upper porous sets by $\mathbf{U P}$. Observe that $\mathbf{S P}_{1}$ is the ideal of countable sets of ${ }^{\omega} 2$. Further research about different types of porosity can be found in Rojas-Rebolledo [16], Zajíček [17], Zapletal [18], Zelený [20] and Zelený and Zajíček [21].

Cardinal invariants of these $\sigma$-ideals have been studied in Brendle [3], Hrušák and Zindulka [6] and Repický $[13,14,15]$. Recall that, given a $\sigma$-ideal $\mathcal{I}$ over a set $X$, the following are the standard cardinal invariants of $\mathcal{I}$ :

$$
\begin{aligned}
\operatorname{add}(\mathcal{I}) & =\min \{|\mathcal{A}|: \mathcal{A} \subseteq \mathcal{I} \wedge \bigcup \mathcal{A} \notin \mathcal{I}\} \\
\operatorname{cov}(\mathcal{I}) & =\min \{|\mathcal{A}|: \mathcal{A} \subseteq \mathcal{I} \wedge \bigcup \mathcal{A} \neq X\} \\
\operatorname{non}(\mathcal{I}) & =\min \{|Y|: Y \subseteq X \wedge Y \notin \mathcal{I}\} \\
\operatorname{cof}(\mathcal{I}) & =\min \{|\mathcal{A}|: \mathcal{A} \subseteq \mathcal{I} \wedge \forall B \in \mathcal{I}(\exists A \in \mathcal{A}(B \subseteq A))\}
\end{aligned}
$$

In [6], Hrušák and Zindulka proved that the cardinal invariants of the $\sigma$-ideal of lower porous sets in the real line are the same as the cardinal invariants of SP. They proved that $\operatorname{non}(\mathbf{S P})<\mathfrak{m}_{\sigma \text {-centered }}$ is consistent, that $\operatorname{cov}(\mathbf{S P})>\operatorname{cof}(\mathcal{N})$ is consistent, and that $\operatorname{cov}(\mathbf{S P})<\mathfrak{c}$ is consistent, where $\mathfrak{m}_{\sigma \text {-centered }}$ is the smallest cardinal where the Martin's axiom for $\sigma$-centered forcings fails and $\mathcal{N}$ is the ideal of sets of Lebesgue measure zero.

There are some analogous inequalities that hold for UP. In [15], M. Repický proved that $\operatorname{non}(\mathbf{U P}) \geq \mathfrak{m}_{\sigma \text {-centered }}$ and $\operatorname{cov}(\mathbf{U P}) \leq \operatorname{cof}(\mathcal{N})$ holds. He proved in [13] that $\operatorname{non}(\mathbf{U P}) \geq \operatorname{add}(\mathcal{N})$ and in [3], J. Brendle proved that $\operatorname{add}(\mathbf{U P})=\omega_{1}$ and $\operatorname{cof}(\mathbf{U P})=\mathfrak{c}$ hold. In [6], Hrušák and Zindulka asked if the last three inequalities hold also for the SP ideal. In this work we show that $\operatorname{add}(\mathbf{S P})=\omega_{1}, \operatorname{cof}(\mathbf{S P})=\mathfrak{c}$ and that it is consistent that $\operatorname{non}(\mathbf{S P})<\operatorname{add}(\mathcal{N})$.

Given $k \in \omega$ and a forcing notion $\mathbb{P}$ a subset $A \subseteq \mathbb{P}$ is $k$-linked if for every collection $\left\{a_{i}: i \in k\right\}$ of $k$ elements of $A$, there is an $a \in \mathbb{P}$ such that for every $i \in k, a \leq a_{i} . \mathbb{P}$

\footnotetext{
${ }^{2} \sigma$-ideals are ideals closed under countable unions of their elements.
} 
is $\sigma$-k-linked if $\mathbb{P}$ is the countable union of $k$-linked subsets of $\mathbb{P}$. We will denote by $\mathfrak{m}_{k}$ the Martin number for $\sigma$ - $k$-linked forcings, that is, the smallest cardinal $\kappa$ such that there is a $\sigma-k$-linked forcing $\mathbb{P}$ and $\kappa$ many $\mathbb{P}$-dense subsets of $\mathbb{P}$ such that no filter of $\mathbb{P}$ intersects them all. In [4], Brendle and Shelah constructed a model where all the Martin numbers of the form $\mathfrak{m}_{2^{i}}$ are distinct. We will show a connection between $\sigma-k$-linked forcings and porous sets and we will use this connection to construct a model where all the Martin numbers $\mathfrak{m}_{i}$ are different all at once.

If $X, Y$ are sets, then ${ }^{Y} X$ is the set of all functions from $Y$ to $X$ and ${ }^{<\omega} X=\bigcup_{n \in \omega}{ }^{n} X$. If $\sigma, s \in{ }^{<\omega} X$, then we will denote that $\sigma$ is an initial segment of $s$ by $\sigma \sqsubseteq s$. A set $T \subseteq{ }^{<\omega} X$ is a tree if it is closed under restrictions to initial segments. If $T \subseteq{ }^{<\omega} X$ is a tree, then by $[T]$ we denote the set of branches of $T$, that is, $[T]=\left\{f \in{ }^{\omega} X: \forall n \in\right.$ $\omega(f \nmid n \in T)\}$. With end $(T)$ we will denote the end nodes of $T$, that is the nodes of $T$ without extensions. In our forcing notation, the stronger conditions are the smaller ones. In general, our notation follows Bartoszyński and Judah [1].

\section{Additivity and cofinality}

The main goal of this section is to prove that $\operatorname{add}(\mathbf{S P})=\omega_{1}$ and $\operatorname{cof}(\mathbf{S P})=\mathfrak{c}$. We will use the following notion.

Definition 2.1 Let $k \in \omega$. A tree $T \subseteq<\omega_{2}$ is a $k$-porous tree if for every $s \in<\omega_{2}$ there is $t \in{ }^{k} 2$ such that $s^{\wedge} t \notin T$.

Note that $A \subseteq{ }^{\omega} 2$ is $k$-porous if and only if there is a $k$-porous tree $T$ such that $[T]$ contains $A$.

Theorem 2.2 There is a family $\left\{T_{f}: f \in{ }^{\omega} 2\right\}$ of 2-porous trees such that for every $X \in \mathbf{S P}$, the set $\left\{f \in{ }^{\omega} 2:\left[T_{f}\right] \subseteq X\right\}$ is countable.

Proof We will construct the family $\left\{T_{f}: f \in \omega^{\omega}\right\}$ as follows: For every $a \subseteq{ }^{<\omega_{2}}$ such that $|a|=2^{n}$, let $\varphi_{a}: a \rightarrow{ }^{n} 2$ be a bijective function. For every $i \in \omega$, let $\psi_{i}:\left\{a \subseteq{ }^{i} 2: \exists k \in \omega\left(|a|=2^{k}\right)\right\} \rightarrow \omega \backslash\{0\}$ be an injective function. If $a \subseteq{ }^{i} 2$ and $|a|=2^{k}$, define

$$
\sigma_{a}=\langle 0, \underbrace{1, \ldots, 1}_{2 \psi_{i}(a) \text { times }}, 0\rangle .
$$


For each $\sigma \in<\omega_{2}$, we will recursively define a finite tree $T_{\sigma}$ as follows: $T_{\emptyset}=\{\emptyset\}$, and if $T_{\sigma}$ is defined then

$$
\begin{aligned}
& T_{\sigma^{\wedge} i}=\left\{s \in{ }^{<\omega} 2: \exists t \in \operatorname{end}\left(T_{\sigma}\right)(\exists j \in \omega(\exists a \subseteq|\sigma|+12\right. \\
& \left.\left.\left.\left(|a|=2^{j} \wedge \sigma^{\frown} i \in a \wedge s \sqsubseteq t^{\wedge} \sigma_{a}^{\wedge} \varphi_{a}\left(\sigma^{\frown} i\right)\right)\right)\right)\right\} \\
& \cup\left\{s \in{ }^{<\omega} 2: \exists t \in \operatorname{end}\left(T_{\sigma}\right)\left(s \sqsubseteq t^{\wedge}\langle 1,1\rangle\right)\right\} .
\end{aligned}
$$

It is easy to see that, for each $\sigma \in{ }^{<\omega} 2, T_{\sigma}$ is a finite 2-porous tree and that if $\sigma \sqsubseteq \tau$, then $T_{\sigma} \subseteq T_{\tau}$. For each $f \in{ }^{\omega} 2$, define $T_{f}=\bigcup_{n \in \omega} T_{f n}$. It follows easily that each $T_{f}$ is a 2 -porous tree.

We will show that the family $\left\{T_{f}: f \in^{\omega} 2\right\}$ is the family we were looking for: Let $X \in \mathbf{S P}$. Without loss of generality we will assume that $X=\bigcup_{i \in \omega}\left[T_{i}\right]$, where $T_{i}$ is an $i+1$-porous tree. We must show that the set $B=\left\{f \in \omega_{2}:\left[T_{f}\right] \subseteq X\right\}$ is countable: For each $s, t \in{ }^{<\omega} 2$ and each $n \in \omega$, define $B_{s, t, n}=\left\{f \in{ }^{\omega} 2: t \sqsubseteq f, s \in\right.$ $\left.T_{t} \wedge\left[T_{f}\right] \cap\langle s\rangle \subseteq\left[T_{n}\right]\right\}$. We will see that $B \subseteq \bigcup_{s, t \in<\omega_{2, n \in \omega} B_{s, t, n}}$ : If $f$ is such that $f \in B$, then $\left[T_{f}\right] \subseteq \bigcup_{n \in \omega}\left[T_{n}\right]$. Using the Baire Category Theorem we can find $s \in T_{f}$ and $n \in \omega$ such that $\left[T_{f}\right] \cap\langle s\rangle \subseteq\left[T_{n}\right]$. Find $k \in \omega$ such that $s \in T_{f k}$. It follows that $f \in B_{s, f k, n}$. To finish the proof we will see that each $B_{s, t, n}$ has at most $2^{n+1}-1$ elements: Suppose this is not the case and let $s, t \in{ }^{<\omega} 2, n \in \omega$ and $\left\{f_{i}\right\}_{i<2^{n+1}} \subseteq B_{s, t, n}$. Extend $s$ to $\sigma$ such that $\sigma \in \operatorname{end}\left(T_{t}\right)$. Let $j \in \omega$ be such that the set $\left.a=\left\{f_{i}\right\rceil j: i<2^{n+1}\right\}$ has $2^{n+1}$ elements and let

$$
\left.s_{0}=\sigma_{2 \cdot(j-|t|-1) \text { times }}^{\langle\underbrace{1, \ldots, 1}}\right\rangle^{\curvearrowright} \sigma_{a} .
$$

The tree $T_{n}$ is $n+1$-porous, so there is a $\tau \in 2^{n+1}$ such that $s_{0} \tau \notin T_{n}$. Find $k<2^{n+1}$ such that $\varphi_{a}\left(f_{k} \uparrow j\right)=\tau$ and observe that $\widehat{s_{0}} \tau=\widehat{s_{0}} \varphi_{a}\left(f_{k} \uparrow j\right) \in T_{f_{k}}$. As a consequence, $\left[T_{f_{k}}\right] \cap\langle s\rangle \nsubseteq\left[T_{n}\right]$, but this contradicts the fact that $f_{k} \in B_{s, t, n}$. This implies that each $B_{s, t, n}$ is finite, and therefore $B$ is countable.

We can now prove the main result of this section.

Corollary 2.3 $\operatorname{add}(\mathbf{S P})=\omega_{1}, \operatorname{cof}(\mathbf{S P})=\mathfrak{c}$.

Proof Let $\left\{T_{f}: f \in{ }^{\omega} 2\right\}$ be the family given by the theorem above. If $H \subseteq{ }^{\omega} 2$ is an uncountable set, then $\bigcup\left\{\left[T_{f}\right]: f \in H\right\} \notin \mathbf{S P}$. As a consequence, $\operatorname{add}(\mathbf{S P})=\omega_{1}$. On the other hand, if $\kappa<\mathfrak{c}$ and if $\left\{X_{\alpha}: \alpha<\kappa\right\} \subseteq \mathbf{S P}$, then there is an $f \in{ }^{\omega} 2$ such that, for every $\alpha<\kappa,\left[T_{f}\right] \nsubseteq X_{\alpha}$ and therefore $\operatorname{cof}(\mathbf{S P})=\mathfrak{c}$. 
Observe that this last proof can be used to show that $\operatorname{add}\left(\mathbf{S P} \mathbf{P}_{n}\right)=\omega_{1}=\operatorname{add}(\mathbf{S P})$ and $\operatorname{cof}\left(\mathbf{S} \mathbf{P}_{n}\right)=\mathfrak{c}=\operatorname{cof}(\mathbf{S P})$.

\section{Uniformity number}

In this section we will study some properties concerning the uniformity number of porosity ideals. We will prove the consistency of $\operatorname{non}(\mathbf{S P})<\operatorname{add}(\mathcal{N})$. We will also develop some tools that we will use later in the paper. We will need the following concept, inspired by the concept of a $k$-Sacks tree in ${ }^{<\omega} k$.

Definition 3.1 Let $k>1$. A tree $T \subseteq{ }^{<\omega} k$ is a $k$-anti-Sacks tree if for every $s \in T$ there is $i<k$ such that $s^{\wedge}\langle i\rangle \notin T$. We will denote by $\mathbf{A S}_{k}$ the $\sigma$-ideal generated by the branches of $k$-anti-Sacks trees.

This notion is the analogue of the notion of 1 -porous tree in ${ }^{<\omega} k$ and it is closely related to the $k$-Sacks forcing. Recall that a $k$-Sacks tree $T$ is a tree on ${ }^{<\omega} k$ such that for every $s \in T$, there is a $t \in T$ such that, for every $i<k, t^{\frown} i \in T$. The $k$-Sacks forcing $\mathbb{S}_{k}$ is the collection of all $k$-Sacks trees ordered by reverse inclusion. It is well-known that the $k$-Sacks forcing is equivalent to $\operatorname{Borel}\left({ }^{\omega} k\right) / \mathbf{A S}_{k}$ (see Newelski and Rosłanowski [9]).

Using a similar argument to the one we gave in the last section, it is possible to show that $\operatorname{add}\left(\mathbf{A} \mathbf{S}_{k}\right)=\omega_{1}$ and that $\operatorname{cof}\left(\mathbf{A} \mathbf{S}_{k}\right)=\mathfrak{c}$. Alternatively, a proof of this fact can be found in [9].

The ideals $\mathbf{S P}_{k}$ and $\mathbf{A} \mathbf{S}_{2^{k}}$ share many properties. Many of the results in this work will concern properties of the ideals $\mathbf{A S}_{k}$ that are also valid for the ideals $\mathbf{S} \mathbf{P}_{k}$, and the proofs for both ideals are almost the same. Whenever we state a property about one of these ideals that is also valid for the other one, we will only give the proof for the ideal $\mathbf{A S}_{k}$.

We shall introduce a notion that we will use to keep the uniformity number small in a forcing extension.

Definition 3.2 Let $\mathbb{P}$ be a forcing notion and let $A \subseteq{ }^{\omega} k$ ( $\left.A \subseteq{ }^{\omega} 2\right)$ be such that $A \notin \mathbf{A} \mathbf{S}_{k}\left(A \notin \mathbf{S P}_{k}\right)$. We say that $\mathbb{P}$ strongly preserves non $\left(\mathbf{A} \mathbf{S}_{k}\right)$ in $A$ ( $\mathbb{P}$ strongly preserves non $\left(\mathbf{S P}_{k}\right)$ in $\left.A\right)$ if for every $\mathbb{P}$-name $\dot{X}$ of a $k$-anti-Sacks tree ( $k$-porous tree) there is a $Y \in \mathbf{A S}_{k}\left(Y \in \mathbf{S P}_{k}\right)$ such that, for every $x \in A$, if $x \notin Y$ then $\mathbb{P} \Vdash$ " $x \notin[\dot{X}]$ ". We will say that $\mathbb{P}$ strongly preserves non $\left(\mathbf{A S}_{k}\right)\left(\mathbb{P}\right.$ strongly preserves non $\left.\left(\mathbf{S P}_{k}\right)\right)$ if $\mathbb{P}$ strongly preserves non $\left(\mathbf{A} \mathbf{S}_{k}\right)$ in ${ }^{\omega} k\left(\mathbb{P}\right.$ strongly preserves non $\left(\mathbf{S P}_{k}\right)$ in $\left.{ }^{\omega} 2\right)$. 
It is easy to see that, if $\mathbb{P}$ strongly preserves non $\left(\mathbf{A} \mathbf{S}_{k}\right)$ in $A$, then $\mathbb{P} \Vdash ~ " A \notin \mathbf{A} \mathbf{S}_{k}$ " and if $\mathbb{P}$ strongly preserves non $\left(\mathbf{A} \mathbf{S}_{k}\right)$, then $\mathbb{P}$ strongly preserves non $\left(\mathbf{A} \mathbf{S}_{k}\right)$ in $A$ for every $A \subseteq{ }^{\omega} k$. The following lemma is easy to prove.

Lemma 3.3 Suppose that a forcing notion $\mathbb{P}$ strongly preserves non $\left(\mathbf{S} \mathbf{P}_{k}\right)$ for every $k \in \omega$, then $\mathbb{P} \Vdash$ " $\omega 2 \cap V \notin \mathbf{S P}$ ”.

Proof The proof is straightforward from the definitions.

The next lemma shows that there is a connection between anti-Sacks trees and $\sigma-k$-linked forcings.

Lemma 3.4 Let $\mathbb{P}$ be a forcing notion. If $\mathbb{P}$ is $\sigma-k$-linked, then $\mathbb{P}$ strongly preserves $\operatorname{non}\left(\mathbf{A} \mathbf{S}_{k}\right)$.

Proof Let $\left\{\mathbb{P}_{i}: i \in \omega\right\}$ be a sequence of $k$-linked subsets such that $\mathbb{P}=\bigcup_{i \in \omega} \mathbb{P}_{i}$. Let $\dot{A}$ be a $\mathbb{P}$-name of a $k$-anti-Sacks tree. Define $T_{n} \subseteq{ }^{\omega} k$ as follows:

$$
T_{n}=\left\{s \in{ }^{<\omega} k: \exists p \in \mathbb{P}_{n}(p \Vdash \text { " } s \in \dot{A} ")\right\}
$$

We claim that, for each $n \in \omega, T_{n}$ is a $k$-anti-Sacks tree. Suppose this is not the case, so there is an $s \in T_{n}$ such that, for every $i \in k, s^{\wedge} i \in T_{n}$. For every $i \in k$, we can pick a condition $p_{i} \in \mathbb{P}_{n}$ such that $p_{i} \Vdash$ " $\left.s\right\urcorner i \in \dot{A}$ ". Let $p \in \mathbb{P}$ be such that, for every $i \in k$, $p \leq p_{i}$. Then $p \Vdash ~ " \forall i \in k\left(s^{\frown} i \in \dot{A}\right)$ ". This contradicts the fact that $\dot{A}$ is a $\mathbb{P}$-name of a $k$-anti-Sacks tree.

To conclude the proof, note that for every $x \in{ }^{\omega} k$, if $p \Vdash$ " $x \in[\dot{A}]$ ", then $x \in\left[T_{n}\right]$, where $n$ is such that $p \in \mathbb{P}_{n}$.

The lemma above is optimal in the sense that, for each $k$, there is a $\sigma-(k-1)$-linked forcing $\mathbb{P}_{k}$ such that $\mathbb{P}_{k} \Vdash " \omega \omega \cap V \in \mathbf{A S}_{k}$ " and therefore $\mathbb{P}_{k}$ does not strongly preserve $\mathbf{A S}_{k}$. This will be proved in the next section. There is also a relation between porous sets in ${ }^{\omega} 2$ and $\sigma$-k-linked forcings.

Lemma 3.5 Let $\mathbb{P}$ be a forcing notion. If $\mathbb{P}$ is $\sigma-2^{k}$-linked, then $\mathbb{P}$ strongly preserves $\operatorname{non}\left(\mathbf{S P}_{k}\right)$.

Proof The proof is similar to the previous lemma. 
We shall show that strong preservation of non $\left(\mathbf{A S}_{k}\right)$ and non $\left(\mathbf{S P}_{k}\right)$ is preserved by finite support iterations.

Lemma 3.6 Let $A \subseteq{ }^{\omega} k$ and $\mathcal{I} \in\left\{\mathbf{S P}_{n}, \mathbf{A S}_{k}: n>0, k>1\right\}$.

(1) If $\mathbb{P}$ is a forcing notion such that $\mathbb{P}$ strongly preserves non $(\mathcal{I})$ in $A$ and $\dot{\mathbb{Q}}$ is a $\mathbb{P}$-name for a forcing such that $\mathbb{P} \Vdash$ “Q $\operatorname{Q}$ strongly preserves non $(\mathcal{I})$ in $A$ ”, then $\mathbb{P} * \dot{\mathbb{Q}}$ strongly preserves non $(\mathcal{I})$ in $A$.

(2) If $\left\langle\mathbb{P}_{\alpha}, \dot{\mathbb{Q}}_{\alpha}: \alpha \leq \gamma\right\rangle$ is a finite support iteration of c.c.c. forcings such that $\mathbb{P}_{\alpha} \Vdash “ \dot{\mathbb{Q}}_{\alpha}$ strongly preserves non $(\mathcal{I})$ in $A$ ” for each $\alpha \in \gamma$, then $\mathbb{P}_{\gamma}$ strongly preserves non $(\mathcal{I})$ in $A$.

Proof We will only show the case when $\mathcal{I}=\mathbf{A S}_{k}$ as the other cases are similar. The part (1) is easy, we will proceed with part (2) by induction on $\gamma$. It is easy to see that the lemma holds for successor ordinals, and if $\gamma$ has uncountable cofinality we can use a standard reflection argument to show that $\mathbb{P}_{\gamma}$ strongly preserves non $\left(\mathbf{A S}_{k}\right)$ in $A$, so it is enough to show that the lemma holds for $\gamma=\omega$. Let $\dot{T}$ be a $\mathbb{P}_{\omega}$-name of a $k$-anti-Sacks tree. For each $n \in \omega$, let $\dot{T}_{n}$ be a $\mathbb{P}_{n}$-name for the following set:

$$
\dot{T}_{n}=\left\{s \in{ }^{<\omega} k: \mathbb{P}_{(n, \omega)} \Vdash “ s \in \dot{T} "\right\}
$$

It is easy to see that each $\dot{T}_{n}$ is a $\mathbb{P}_{n}$ name for a $k$-anti-Sacks tree. Now we use that each $\mathbb{P}_{n}$ strongly preserves non $\left(\mathbf{A S}_{k}\right)$ to find a family $\left\{T_{i}^{j}: i, j \in \omega\right\}$ such that, for each $n \in \omega$, if $x \in A$ and $x \notin \bigcup_{i \in \omega}\left[T_{i}^{n}\right]$, then $\mathbb{P}_{n} \Vdash$ " $x \notin\left[\dot{T}_{n}\right]$ ". It is easy to see that the set $Y=\bigcup\left\{\left[T_{i}^{j}\right]: i, j \in \omega\right\}$ is the set we are looking for.

We will now prove the consistency of $\operatorname{non}(\mathbf{S P})<\operatorname{add}(\mathcal{N})$. For constructing the model we are looking for, we will use the amoeba forcing $\mathbb{A}$ in the following presentation:

$$
\mathbb{A}=\left\{B \in \operatorname{Borel}\left({ }^{\omega} 2\right): \mu(B)>\frac{1}{2}\right\}
$$

Here Borel $\left({ }^{\omega} 2\right)$ represents the collection of Borel subsets of the Cantor space and $\mu$ is the standard Lebesgue measure over ${ }^{\omega} 2$. The order is given by $A \leq B$ if and only if $A \subseteq B$. The following lemma is well-known (see eg Bartoszyński and Judah[1]). We include the simple proof for the sake of completeness.

Lemma 3.7 The amoeba forcing is $\sigma$-n-linked for every $n \in \omega$. 
Proof Let $n \in \omega$. For every clopen $C$ in ${ }^{\omega} 2$, define

$$
\mathbb{A}_{C}=\left\{A \in \mathbb{A}: \mu(C \backslash A)<\frac{1}{n} \cdot\left(\mu(C)-\frac{1}{2}\right)\right\} .
$$

We will show that $\mathbb{A}=\bigcup\left\{\mathbb{A}_{C}: C\right.$ is a clopen in $\left.{ }^{\omega} 2\right\}$. Let $A \in \mathbb{A}$ and let $\varepsilon>0$ such that $\mu(A)=\frac{1}{2}+\varepsilon$. Find an open set $U \subseteq{ }^{\omega} 2$ such that $A \subseteq U$ and $\mu(U \backslash A)<\frac{\varepsilon}{n}$. Now find a clopen set $C \subseteq U$ such that $\mu(C)>\frac{1}{2}+\varepsilon$. Then

$$
\mu(C \backslash A)<\mu(U \backslash A)<\frac{\varepsilon}{n}=\frac{1}{n} \cdot\left(\frac{1}{2}+\varepsilon-\frac{1}{2}\right)<\frac{1}{n} \cdot\left(\mu(C)-\frac{1}{2}\right) .
$$

Therefore $A \in \mathbb{A}_{C}$. Now we must show that, for every clopen set $C \subseteq{ }^{\omega} 2$, the intersection $K$ of an arbitrary family $\left\{A_{j}: j \in n\right\} \subseteq \mathbb{A}_{C}$ is an element of $\mathbb{A}$. This is a consequence of the following calculations:

$$
\mu(C) \leq \mu(K)+\sum_{j \in n} \mu\left(C \backslash A_{j}\right)<\mu(K)+\frac{1}{n} \cdot\left(\sum_{j \in n} \mu(C)-\frac{1}{2}\right)=\mu(K)+\mu(C)-\frac{1}{2} .
$$

As a consequence, $\frac{1}{2}<\mu(K)$. Therefore $K \in \mathbb{A}$.

We are ready to prove the main result of this section. The method of the proof was suggested to us by J. Brendle.

Theorem 3.8 If ZFC is consistent, then ZFC $+\operatorname{non}(\mathbf{S P})<\operatorname{add}(\mathcal{N})$ is consistent.

Proof Start with a model $V$ such that $V=C H$. Let $\mathbb{P}=\left\{\mathbb{P}_{\alpha}, \dot{\mathbb{Q}}_{\alpha}: \alpha<\omega_{2}\right\}$ be a finite support iteration of the amoeba forcing. It follows from the lemmas above that $\mathbb{P}$ strongly preserves non $\left(\mathbf{S P}_{k}\right)$ for every $k \in \omega$ and therefore $\mathbb{P} \Vdash$ " $\omega 2 \cap V \notin \mathbf{S P}$ ". As a consequence, we have that $V[G]=\operatorname{non}(\mathbf{S P})=\omega_{1}$. It is a known fact (see [1]) that $V[G] \models \operatorname{add}(\mathcal{N})=\omega_{2}$, hence $V[G] \models \operatorname{non}(\mathbf{S P})<\operatorname{add}(\mathcal{N})$.

\section{Martin numbers of $\sigma-k$-linked forcings}

It is easy to see that $\mathfrak{m}_{2} \leq \mathfrak{m}_{3} \leq \ldots$ and, for each $k>1$, it is possible to get the consistency of $\mathfrak{m}_{k}<\mathfrak{m}_{k+1}$ by forcing with a finite support iteration of $\sigma-(k+1)$-linked forcings over a model of $\mathrm{CH}$. In [4], Brendle and Shelah constructed a model where all the cardinals of the form $\mathfrak{m}_{2^{k}}$ are different. In this section we will construct a model where all the Martin numbers $\mathfrak{m}_{i}$ are different at the same time. In this model, the cardinals non $\left(\mathbf{A S}_{i}\right)$ will be different all at once (so will be the cardinals non $\left(\mathbf{S P}_{i}\right)$ ). We will use the following forcing notions. Given $k>2$ let 


$$
\begin{aligned}
\mathbb{P}_{k}=\{\langle s, F\rangle: & \text { (a) } s \text { is a finite } k \text {-anti-Sacks tree of height } h t(s), \\
& \text { (b) } F \in\left[{ }^{\omega} k\right]^{<\omega}, \text { and }\left\lceil F\left\lceil\Delta_{F}\right\rceil \text { is a finite } k\right. \text {-anti-Sacks tree, and } \\
& \text { (c) } s \subseteq\left\lceil F\left\lceil\Delta_{F}+1\right\rceil\right\},
\end{aligned}
$$

where $F\left\lceil k=\left\{f\lceil k: f \in F\},\lceil F\rceil=\left\{\sigma \in{ }^{<\omega} k: \exists f \in F(\sigma \subseteq f)\right\}\right.\right.$ and $\Delta_{F}=\min \{n \in$ $\omega:|F| n|=| F \mid\}$. The order is defined by $\left\langle s^{\prime}, F^{\prime}\right\rangle \leq\langle s, F\rangle$ if and only if $s \subseteq s^{\prime}$ and $F \subseteq F^{\prime}$. This forcing notion will be used to work with the ideal $\mathbf{A S}_{k}$. For the ideal $\mathbf{S P}_{k}$, we will be using a similar forcing notion. Given $k>1$ let

$$
\begin{aligned}
\mathbf{P}_{k}=\{\langle s, F\rangle: & (a) s \text { is a finite } k \text {-porous tree, and } \\
& \left(\text { b) } F \in [ { } ^ { \omega } 2 ] ^ { < \omega } \text { , and } \left\lceilF\left\lceil\Delta_{F}\right\rceil \text { is a finite } k\right.\right. \text {-anti-Sacks tree, and } \\
& \text { (c) } s \subseteq\left\lceil F\left\lceil\Delta_{F}+1\right\rceil\right\} .
\end{aligned}
$$

The order is defined by $\left\langle s^{\prime}, F^{\prime}\right\rangle \leq\langle s, F\rangle$ if and only if $s \subseteq s^{\prime}$ and $F \subseteq F^{\prime}$. We will be using the following proposition.

Proposition 4.1 Given a $k>2$ and an $i>1, \mathbb{P}_{k} \Vdash{ }^{"}{ }^{\omega} k \cap V \in \mathbf{A S}_{k}$ " and $\mathbf{P}_{i} \Vdash " \omega 2 \cap V \in \mathbf{S P}_{i} "$.

Proof We will only check that $\mathbb{P}_{k} \Vdash " \omega k \cap V \in \mathbf{A S}_{k}$ " as the other part is similar. It is easy to see that, for every $f \in{ }^{\omega} k$ and $n \in \omega$, the following sets are dense in $\mathbb{P}$ :

$$
\begin{aligned}
& D_{f}=\left\{\langle s, F\rangle \in \mathbb{P}_{k}: \exists \sigma \in{ }^{<\omega} k\left(\sigma^{\frown} f \uparrow(\omega \backslash|\sigma|) \in F\right)\right\} \\
& E_{n}=\left\{\langle s, F\rangle \in \mathbb{P}_{k}: \Delta_{F}>n \wedge s=\left\lceil F \uparrow \Delta_{F}+1\right\rceil\right\}
\end{aligned}
$$

If $G \subseteq \mathbb{P}_{k}$ is a filter meeting all these dense sets, then, using that the sets $E_{n}$ are dense, it follows that $T=\bigcup\{s: \exists F(\langle s, F\rangle \in G)\}$ is a $k$-anti-Sacks tree. If $\sigma \in{ }^{<\omega} k$ and if $C[\sigma]=\left\{\sigma^{\curlyvee} x \uparrow(\omega \backslash|\sigma|): x \in[T]\right\}$, then, using that the $D_{f}$ are dense, it follows that ${ }^{\omega} k \cap V \subseteq \bigcup\left\{C[\sigma]: \sigma \in{ }^{<\omega} k\right\} \in \mathbf{A S}_{k}$.

The last proposition together with the Lemma 3.4 implies that $\mathbb{P}_{k}$ is not $\sigma$-k-linked. In contrast with this last observation, we have the following proposition.

Proposition 4.2 For each $k>1, \mathbb{P}_{k+1}$ is $\sigma-k$-linked and $\mathbf{P}_{k}$ is $\sigma-\left(2^{k}-1\right)$-linked. 
Proof Again, we will only check that $\mathbb{P}_{k+1}$ is $\sigma-k$-linked, the other part is done in a similar way: For any two finite $(k+1)$-anti-Sacks trees $s, t$ of height $h t(s), \operatorname{ht}(t)$ respectively, define

$$
P(s, t)=\left\{\langle s, F\rangle \in \mathbb{P}_{k+1}: \operatorname{ht}(t)>\Delta_{F} \wedge F\lceil\operatorname{ht}(t)=t\} .\right.
$$

It is easy to see that $\mathbb{P}_{k+1}=\bigcup\{P(s, t): s, t$ are finite $(k+1)$-anti-Sacks trees $\}$. We will show that every $P(s, t)$ is $k$-linked. Let $\left\{\left\langle s, F_{i}\right\rangle: i<k\right\} \subseteq P(s, t)$ and let $F=\bigcup_{i<k} F_{i}$. We must show that $\langle s, F\rangle \in \mathbb{P}_{k+1}$. The properties (a) and (c) are easily verified, so the only thing left to do is to show that $\left\lceil F\left\lceil\Delta_{F}\right\rceil\right.$ is a $(k+1)$-anti-Sacks tree. Let $\sigma \in\left\lceil F\left\lceil\Delta_{F}\right\rceil\right.$. If $|\sigma|<\mathrm{ht}(t)$, then, because $F\lceil\mathrm{ht}(t)=t$, it is possible to find an $i \in k$ such that $\sigma^{\wedge}\langle i\rangle \notin\left\lceil F\left\lceil\Delta_{F}+1\right\rceil\right.$. If $|\sigma| \geq \mathrm{ht}(t)$, then, for every $i<k, \sigma$ only has (at most) one immediate successor in $F_{i}$ and therefore it is always possible to find a $j \in k+1$ such that $\sigma^{\frown}\langle j\rangle \notin\left\lceil F\left\lceil\Delta_{F}+1\right\rceil\right.$.

From these last two propositions we get the following result.

Corollary 4.3 For each $k>1, \mathfrak{m}_{k} \leq \operatorname{non}\left(\mathbf{A S}_{k+1}\right)$ and $\mathfrak{m}_{2^{k}-1} \leq \operatorname{non}\left(\mathbf{S P}_{k}\right)$

Proof This follows easily from the proof of the Proposition 4.1 and the last proposition.

For the proof of the main theorem we will need the following notion.

Definition 4.4 Given a regular cardinal $\kappa$ and $\mathcal{I} \in\left\{\mathbf{S P}_{n}, \mathbf{A S}_{k}: n>0, k>1\right\}$, we will say that a set $L$ is $\langle\kappa, \mathcal{I}\rangle$-Luzin if $|L|=\kappa$ and $\mathcal{I} \nmid L=[L]^{<\kappa}$.

Observe that the existence of a $\langle\kappa, \mathcal{I}\rangle$-Luzin set implies that non $(\mathcal{I}) \leq \kappa$. Recall that Cohen reals are added at every limit step of countable cofinality of a finite support iteration of arbitrary length. One common application of Cohen reals is that they are used to construct Luzin sets with special properties. The following lemma is one of those applications.

Lemma 4.5 Let $\kappa$ be a regular cardinal, let $i>2, k>1$ and let $\mathbb{L}=\left\langle\mathbb{L}_{\alpha}, \dot{\mathbb{Q}}_{\alpha}: \alpha \in \kappa\right\rangle$ be a finite support iteration of length $\kappa$ such that $\mathbb{L}_{\alpha} \Vdash$ " $\dot{\mathbb{Q}}_{\alpha}=\mathbb{P}_{i} * \mathbf{P}_{k}$ ", then $\mathbb{L} \Vdash$ "There is a $\left\langle\kappa, \mathbf{A S}_{i}\right\rangle$-Luzin set and there is a $\left\langle\kappa, \mathbf{S P}_{k}\right\rangle$-Luzin set". 
Proof Working in $V[G]$, let $L=\left\{f_{\alpha}: \alpha \in \kappa \wedge \alpha\right.$ has countable cofinality $\}$ be a family of Cohen reals such that each $f_{\alpha}$ is added at the $\alpha$-th stage of the iteration. Using the Proposition 4.1, it is easy to show that $V[G]=[L]^{<\kappa} \subseteq \mathbf{A S}_{i} \mid L$. On the other hand, if $T \in V[G]$ is such that $V[G] \models T$ is an $i$-anti-Sacks tree, then, by a standard reflection argument, there is an intermediate model such that $T \in V[G(\beta)]$. As a consequence, $V[G] \models \forall \gamma>\beta\left(f_{\gamma} \notin[T]\right)$. This implies that $V[G] \models \mathbf{A S}_{i} \mid L \subseteq[L]^{<\kappa}$. The $\left\langle\kappa, \mathbf{S P}_{k}\right\rangle$-Luzin set is found in a similar way.

In the lemma above, it is clear that if we replace $\mathbb{L}_{\alpha} \Vdash$ " $\dot{\mathbb{Q}}_{\alpha}=\mathbb{P}_{i} * \mathbf{P}_{k}$ " by $\mathbb{L}_{\alpha} \Vdash " \dot{\mathbb{Q}}_{\alpha}=\mathbb{P}_{i}$ ", then, in the extension, we still have a $\left\langle\kappa, \mathbf{A} \mathbf{S}_{i}\right\rangle$-Luzin set. The following theorem is the main tool we will use to prove the main result of this section.

Theorem 4.6 If ZFC is consistent, then ZFC $+\forall i>2\left(\exists L_{i}\left(L_{i}\right.\right.$ is $\left.\left.\left\langle\aleph_{i}, \mathbf{A S}_{i}\right\rangle-L u z i n\right)\right)+$ $\forall k>1\left(\exists L_{k}^{\prime}\left(L_{k}^{\prime}\right.\right.$ is $\left\langle\aleph_{2^{k}}, \mathbf{S P}_{k}\right\rangle$-Luzin $\left.)\right)$ is consistent.

Proof Let $\mathbb{L}=\left\langle\mathbb{L}_{\alpha}, \dot{\mathbb{Q}}_{\alpha}: \alpha \in \omega_{\omega}\right\rangle$ be a finite support iteration of length $\omega_{\omega}$ such that, for each $i>1$ and each $\alpha \in\left[\omega_{i}, \omega_{i+1}\right), \mathbb{L}_{\alpha} \Vdash$ “这 $=\mathbb{P}_{i+1} * \mathbf{Q}_{i+1}$ ", where $\mathbf{Q}_{i+1}=\mathbf{P}_{i+1}$ when $i+1$ is a number of the form $2^{k}+1$ and $\mathbf{Q}_{i+1}=\{\emptyset\}$ in all the other cases (for $\alpha<\omega_{2}, \mathbb{L}_{\alpha} \Vdash$ “这 $=\{\emptyset\}$ ”). We will show that the extension is the model we are looking for. As usual in this work, we will only show that there are $\left\langle\aleph_{i}, \mathbf{A S}_{i}\right\rangle$-Luzin sets for every $i>2$ (the rest can be done in a similar way). Using the lemma above, for each $i>2$, in $V\left[G_{\omega_{i}}\right]$ there is a $\left\langle\aleph_{i}, \mathbf{A S}_{i}\right\rangle$-Luzin set $L_{i}$. The only thing left to do is to show that $L_{i}$ remains $\left\langle\aleph_{i}, \mathbf{A S}_{i}\right\rangle$-Luzin in $V[G]$. Using that $\mathbb{L}$ is c.c.c. it is easy to see that, in $V[G],\left[L_{i}\right]^{<\omega_{i}} \subseteq \mathbf{A S}_{i}\left\lceil L_{i}\right.$, so we only need to show that $\mathbf{A} \mathbf{S}_{i}\left\lceil L_{i} \subseteq\left[L_{i}\right]^{<\omega_{i}}\right.$ holds in $V[G]$. First, using Lemma 3.4, Lemma 3.6 and Proposition 4.2, we observe that $\mathbb{L}_{\left[\omega_{i}, \omega_{\omega}\right]}$ strongly preserves non $\left(\mathbf{A S}_{i}\right)$ in $L_{i}$, so if $\dot{T}$ is a $\mathbb{L}_{\left[\omega_{i}, \omega_{\omega}\right]}$-name of a $i$-anti-Sacks tree, then, in $V\left[G_{\omega_{i}}\right]$, there is a $X \in \mathbf{A} \mathbf{S}_{i}\left\lceil L_{i}\right.$ such that

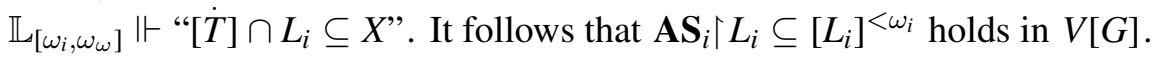

The actual value of $\mathfrak{c}$ in the model above may depend on $V$. For example, if $V \models \mathrm{GCH}$, then it is easy to see that $V[G] \models \mathfrak{c}=\aleph_{\omega+1}$.

Lemma 4.7 Let $\kappa$ be a regular cardinal, let $\mathcal{I} \in\left\{\mathbf{S P}_{n}, \mathbf{A S}_{k}: n>0, k>1\right\}$ and let $L$ be a $\langle\kappa, \mathcal{I}\rangle$-Luzin set. If $\mathbb{P}$ is a forcing notion such that $|\mathbb{P}|<\kappa$, then $\mathbb{P}$ strongly preserves $\operatorname{non}(\mathcal{I})$ in $L$.

Proof We will do the case when $\mathcal{I} \in\left\{\mathbf{A S}_{i}: i>1\right\}$; the other cases are similar. Let $\dot{A}$ be a $\mathbb{P}$-name of an $i$-anti-Sacks tree and let $\mathbb{P}=\left\{p_{\alpha}: \alpha \in \mu\right\}$. For each $\alpha \in \mu$ 
define $T_{\alpha}=\left\{s \in{ }^{<\omega} k: p_{\alpha} \Vdash\right.$ "s $\in \dot{A}$ " $\}$. It follows that each $T_{\alpha}$ defines an $i$-anti-Sacks tree. If $Y=\bigcup\left\{\left[T_{\alpha}\right] \cap L: \alpha \in \mu\right\}$ then $Y \in \mathbf{A S}_{k}$. If $x \in L$ and $p_{\alpha} \Vdash$ " $x \in[\dot{A}]$ ", then $x \in\left[T_{\alpha}\right] \cap L \subseteq Y$.

We are ready to prove the main result of this section.

Theorem 4.8 If ZFC is consistent, then ZFC $+\forall k>1\left(\mathfrak{m}_{k}=\operatorname{non}\left(\mathbf{A S}_{k+1}\right)=\aleph_{k+1}\right)+$ $\forall i>1\left(\mathfrak{m}_{2^{i}-1}=\operatorname{non}\left(\mathbf{S P}_{i}\right)=\aleph_{2^{i}}\right)+\operatorname{non}(\mathbf{S P})=\aleph_{\omega+1}$ is consistent.

Proof Start with a model $V$ like the one constructed in Theorem 4.6 and $V \mid=\mathfrak{c}=\aleph_{\omega+1}$. Using a standard bookkeeping argument, it is possible to construct a finite support iteration $\mathbb{P}$ of length $\omega_{\omega+1}$ of $\sigma$-k-linked forcings of size smaller than $\aleph_{k+1}$ (for every $k>1$ ), such that any partial order which appears in an intermediate model is listed cofinally along the iteration. Now, using Lemmas 3.4, 3.6 and 4.7, it is possible to show that, for every $k>2, \mathbb{P}$ strongly preserves non $\left(\mathbf{A} \mathbf{S}_{k}\right)$ in $L_{k}$. If $G \subseteq \mathbb{P}$ is a generic filter over $V$, then $V[G] \models \operatorname{non}\left(\mathbf{A S}_{k}\right) \leq \aleph_{k}$. We note that, as each small $\sigma$-k-linked forcing appears in an intermediate model in the iteration, we have $V[G] \models \aleph_{k+1} \leq \mathfrak{m}_{k}$. As a consequence $V[G] \models \aleph_{k+1}=\mathfrak{m}_{k}=\operatorname{non}\left(\mathbf{A S}_{k+1}\right)$. Using a similar argument, it is possible to show that, for each $i>1, V[G] \models \aleph_{2^{i}}=\mathfrak{m}_{2^{i}-1}=\operatorname{non}\left(\mathbf{S P}_{i}\right)$. To finish the proof, use the fact that non(SP) does not have countable cofinality and that, for every $n \in \omega, \operatorname{non}\left(\mathbf{S P}_{n}\right) \leq \operatorname{non}(\mathbf{S P})$ to show that $V[G] \mid=\operatorname{non}(\mathbf{S P})=\mathfrak{c}=\aleph_{\omega+1}$.

It follows from $\mathbf{S P}_{1} \subseteq \mathbf{S P}_{2} \subseteq \mathbf{S P}_{3} \subseteq \ldots$ that $\omega_{1}=\operatorname{non}\left(\mathbf{S P}_{1}\right) \leq \operatorname{non}\left(\mathbf{S P}_{2}\right) \leq$ non $\left(\mathbf{S P}_{3}\right) \leq \ldots \leq$ non $(\mathbf{S P})$ and we proved in the theorem above that each inequality can be consistently strict. It is important to remark that none of these numbers is comparable with $\mathfrak{m}_{\sigma \text {-centered }}$. An argument for this can be found in Hrušák and Zindulka [6].

\section{The covering number}

It follows from the fact that $\mathbf{A S}_{2} \subseteq \mathbf{A} \mathbf{S}_{3} \subseteq \ldots$ that $\mathfrak{c}=\operatorname{cov}\left(\mathbf{A S}_{2}\right) \geq \operatorname{cov}\left(\mathbf{A S}_{3}\right) \geq \ldots$ We can show that every pair of these numbers can be consistently different.

Proposition 5.1 Let $k>1$, if ZFC is consistent, then ZFC $+\operatorname{cov}\left(\mathbf{A} \mathbf{S}_{k+1}\right)<\operatorname{cov}\left(\mathbf{A S} \mathbf{S}_{k}\right)$ is consistent. ${ }^{3}$

\footnotetext{
${ }^{3}$ A similar theorem for the ideals $\mathbf{S} \mathbf{P}_{k}$ can be proved using the same argument.
} 
Proof Let $V$ be a model such that $V \models \operatorname{cov}\left(\mathbf{A S}_{k}\right)=\mathfrak{c}=\omega_{2}$. Let $\mathbb{P}$ be a finite support iteration of length $\omega_{1}$ of the $\mathbb{P}_{k+1}$ forcing defined above and let $G \subseteq \mathbb{P}$ be a generic filter over $V$. It follows that $\mathbb{P}$ is an iteration of $\sigma-k$-linked forcing notions and therefore $\mathbb{P}$ strongly preserves non $\left(\mathbf{A S}_{k}\right)$. In $V[G]$, consider the family $C=\left\{V\left[G_{\alpha}\right] \cap{ }^{\omega}(k+1): \alpha<\omega_{1}\right\}$. Using Proposition 4.1, it is easy to show that $V[G] \models C \subseteq \mathbf{A S}_{k+1}$ and $V[G] \models \bigcup C={ }^{\omega}(k+1)$. As a consequence we have that $V[G]=\operatorname{cov}\left(\mathbf{A S}_{k+1}\right)=\omega_{1}$. On the other hand, if $\left\{\dot{T}_{\alpha}: \alpha \in \omega_{1}\right\}$ is a collection of $\mathbb{P}$-names for $k$-anti-Sacks trees, then we can use the fact that $\mathbb{P}$ strongly preserves non $\left(\mathbf{A S}_{k}\right)$ to show that there is a collection $\left\{C_{\alpha}: \alpha \in \omega_{1}\right\} \subseteq \mathbf{A S}_{k}$ such that if $x \in{ }^{\omega} k$ and $x \notin \bigcup\left\{C_{\alpha}: \alpha \in \omega_{1}\right\}$, then $\mathbb{P} \Vdash ~ " x \notin \bigcup_{\alpha \in \omega_{1}}\left[\dot{T}_{\alpha}\right]$ ". This, together with $V \models \operatorname{cov}\left(\mathbf{A S}_{k}\right)>\omega_{1}$, implies that $V[G] \models \operatorname{cov}\left(\mathbf{A} \mathbf{S}_{k+1}\right)<\operatorname{cov}\left(\mathbf{A} \mathbf{S}_{k}\right)$.

An alternative proof of this proposition follows from the results proven in Newelski and Rosłanowski [9]. If $k>1$ then a tree $T \subseteq{ }^{<\omega} \omega$ is a $k$-tree if every $s \in T$ has at most $k$ immediate successors. A forcing notion $\mathbb{P}$ has the $k$-localization property if $\mathbb{P} \Vdash " \forall f \in{ }^{\omega} \omega(\exists T \in V$ (T is a k-tree and $\left.f \in[T])\right)$ ". It is easy to see that if $\mathbb{P}$ has the $k$-localization property, then $\mathbb{P} \Vdash$ "U( $\left.\mathbf{A} \mathbf{S}_{k+1} \cap V\right)={ }^{\omega}(k+1)$ ". Let $\mathbb{S}_{k}=\left\{T \subseteq{ }^{<\omega} k: \forall s \in T(\exists t \in T(\forall i \in k(s \sqsubseteq t \wedge t \wedge i \in T)))\right\}$ be the $k$-Sacks forcing ordered by inclusion. It turns out that $\mathbb{S}_{k}$ is forcing equivalent to Borel $\left({ }^{\omega} k\right) / \mathbf{A} \mathbf{S}_{k}$ and that if $\mathbb{P}$ is the countable support iteration or the countable support product of length $\omega_{2}$ of the forcing $\mathbb{S}_{k}$, then $\mathbb{P}$ has the $k$-localization property (see [9]). As a consequence, in the extension $\operatorname{cov}\left(\mathbf{A} \mathbf{S}_{k+1}\right)=\omega_{1}$ and $\operatorname{cov}\left(\mathbf{A} \mathbf{S}_{k}\right)=\omega_{2}$.

Obviously it is impossible to separate infinitely many of the $\operatorname{cov}\left(\mathbf{S P}_{n}\right)$ at the same time. This suggests the following:

Question 5.2 How many of the $\operatorname{cov}\left(\mathbf{S P}_{n}\right)$ can be separated at the same time?

We do not even know how to separate three of them. Another question we have is the following:

Question 5.3 Is it possible to get the consistency of ZFC + $\forall k \in \omega\left(\operatorname{cov}(\mathbf{S P})<\operatorname{cov}\left(\mathbf{S P}_{k}\right)\right)$ ?

We are also interested in the relationship between $\operatorname{non}(\mathbf{S P})$ and $\operatorname{cov}(\mathbf{S P})$. It follows from the fact that the Cohen forcing is $\sigma$-centered that, in the Cohen model, non $(\mathbf{S P})<$ $\operatorname{cov}(\mathbf{S P})$. However, we do not know if it is possible to construct a model where $\operatorname{non}(\mathbf{S P})>\operatorname{cov}(\mathbf{S P})$.

Question 5.4 Is non $(\mathbf{S P}) \leq \operatorname{cov}(\mathbf{S P})$ ? 
A related question, as to whether non $\left(\mathbf{A} \mathbf{S}_{n}\right) \leq \operatorname{cov}\left(\mathbf{A} \mathbf{S}_{n}\right)$ was asked in [9]. Finally, we would like to discuss about the relation of the cardinal numbers of the ideals $\mathbf{S P}_{k}$ and $\mathbf{A S}_{2^{k}}$. In this work we showed that these ideals share a lot of properties, however we do not know if they share the same cardinal invariants. There is a connection between $2^{k}$ anti-Sacks trees and $k$-porous sets given by the following argument. Let $\varphi_{k}: 2^{k} \rightarrow{ }^{k} 2$ be a bijective function. Let $\psi_{k}:{ }^{\omega}\left(2^{k}\right) \rightarrow{ }^{\omega} 2$ defined by $\psi_{k}(x)=\varphi_{k}(x(0))^{\wedge} \varphi_{k}(x(1))^{\frown} \ldots$ Clearly, if $\psi_{n}(A) \in \mathbf{S P}_{n}$, then $A \in \mathbf{A S}_{2^{n}}$. We do not know if this can be used to show a relation between the cardinal invariants of the ideals $\mathbf{S P}_{k}$ and $\mathbf{A S}_{2^{k}}$.

Question 5.5 Is non $\left(\mathbf{S P}_{k}\right)=\operatorname{non}\left(\mathbf{A S}_{2^{k}}\right)$ ? Is $\operatorname{cov}\left(\mathbf{S P}_{k}\right)=\operatorname{cov}\left(\mathbf{A} \mathbf{S}_{2^{k}}\right)$ ?

\section{Acknowledgements}

The authors would like to thank J. Brendle and J. Cancino for many helpful suggestions and hours of stimulating conversations. The authors would also like to thank the anonymous referees for their insightful corrections and suggestions. The authors were supported by PAPIIT grants IN 102311 and IN 108014. The first-listed author was supported by CONACyT scholarship 420090. The second-listed author was supported by a CONACyT grant 177758 . The third-listed author was supported by CONACyT scholarship 332652.

\section{References}

[1] T Bartoszyński, H Judah, Set theory, A K Peters, Ltd., Wellesley, MA (1995)On the structure of the real line

[2] C L Belna, M J Evans, P D Humke, Symmetric and ordinary differentiation, Proc. Amer. Math. Soc. 72 (1978) 261-267; doi: 10.2307/2042787

[3] J Brendle, The additivity of porosity ideals, Proc. Amer. Math. Soc. 124 (1996) 285-290; doi: 10.1090/S0002-9939-96-02992-9

[4] J Brendle, S Shelah, Evasion and prediction. IV. Strong forms of constant prediction, Arch. Math. Logic 42 (2003) 349-360; doi: 10.1007/s001530200143

[5] E P Dolženko, Boundary properties of arbitrary functions, Izv. Akad. Nauk SSSR Ser. Mat. 31 (1967) 3-14

[6] M Hrušák, O Zindulka, Cardinal invariants of monotone and porous sets, J. Symbolic Logic 77 (2012) 159-173; doi: 10.2178/jsl/1327068697 
[7] J Lindenstrauss, D Preiss, On Fréchet differentiability of Lipschitz maps between Banach spaces, Ann. of Math. (2) 157 (2003) 257-288; doi: 10.4007/annals.2003.157.257

[8] J Lindenstrauss, D Preiss, J Tǐ ser, Fréchet differentiability of Lipschitz functions and porous sets in Banach spaces, volume 179 of Annals of Mathematics Studies, Princeton University Press, Princeton, NJ (2012)

[9] L Newelski, A Rosłanowski, The ideal determined by the unsymmetric game, Proc. Amer. Math. Soc. 117 (1993) 823-831; doi: 10.2307/2159150

[10] D Preiss, L Zajíček, Fréchet differentiation of convex functions in a Banach space with a separable dual, Proc. Amer. Math. Soc. 91 (1984) 202-204; doi: 10.2307/2044627

[11] S Reich, A J Zaslavski, Well-posedness and porosity in best approximation problems, Topol. Methods Nonlinear Anal. 18 (2001) 395-408

[12] D L Renfro, Porosity, nowhere dense sets and a theorem of Denjoy, Real Anal. Exchange $21(1995 / 96)$ 572-581

[13] M Repický, Porous sets and additivity of Lebesgue measure, Real Anal. Exchange 15 (1989/90) 282-298

[14] M Repický, Additivity of porous sets, Real Anal. Exchange 16 (1990/91) 340-343

[15] M Repický, Cardinal invariants related to porous sets, from: "Set theory of the reals (Ramat Gan, 1991)”, Israel Math. Conf. Proc. 6, Bar-Ilan Univ., Ramat Gan (1993) $433-438$

[16] D Rojas-Rebolledo, Using determinacy to inscribe compact non- $\sigma$-porous sets into non- $\sigma$-porous projective sets, Real Anal. Exchange 32 (2006/07) 55-66; url: http://projecteuclid.org/projecteuclid.rae/1184700036

[17] L Zajíček, On $\sigma$-porous sets in abstract spaces, Abstr. Appl. Anal. (2005) 509-534; doi: 10.1155/AAA.2005.509

[18] J Zapletal, Forcing idealized, volume 174 of Cambridge Tracts in Mathematics, Cambridge University Press, Cambridge (2008); doi: 10.1017/CBO9780511542732

[19] A J Zaslavski, Well-posedness and porosity in optimal control without convexity assumptions, Calc. Var. Partial Differential Equations 13 (2001) 265-293; doi: $10.1007 / \mathrm{s} 005260000073$

[20] M Zelený, The Banach-Mazur game and $\sigma$-porosity, Fund. Math. 150 (1996) 197-210

[21] M Zelený, L Zajíček, Inscribing compact non- $\sigma$-porous sets into analytic non- $\sigma$ porous sets, Fund. Math. 185 (2005) 19-39; doi: 10.4064/fm185-1-2

Centro de Ciencas Matemáticas, UNAM, A.P. 61-3, Xangari, Morelia, Michoacán, 58089, México

Centro de Ciencas Matemáticas, UNAM, A.P. 61-3, Xangari, Morelia, Michoacán, 58089, México 
Centro de Ciencas Matemáticas, UNAM, A.P. 61-3, Xangari, Morelia, Michoacán, 58089, México

oguzman@matmor.unam.mx, michael@matmor.unam.mx, arturo@matmor.unam.mx http://matmor.unam.mx/ michael

Received: 7 September $2016 \quad$ Revised: 7 June 2017 